\title{
Natural Qubit Matrix of Primary Elements of Matter
}

\author{
Eugene Machusky \\ ${ }^{1}$ National Technical University of Ukraine "Kyiv Polytechnic Institute", Kyiv, Ukraine
}

\begin{abstract}
The arithmetic limits of natural bit calculations are strictly established. The natural quantum metric system has been developed. Only seven scaling units that generate thirteen invariant values are necessary and sufficient for an accurate estimation and a mutual coordination of the fundamental constants of quantum physics. For the first time, the calibration constants of quantum physics were obtained, calculated and identified analytically with almost an absolute accuracy, which is limited only by a bit capacity of a computer. The basic constants of quantum physics are, in fact, the dynamic parameters of the functional relationships of the transcendental numbers PI and E with natural numbers, which draw a holographic picture of the motion of spherical wave fronts.
\end{abstract}

\section{Introduction}

The first attempt to develop the absolute metric system was made by Gauss in the early nineteenth century. An attempt was unsuccessful due to the inconsistency of parabolic, elliptical and hyperbolic geometry. The inverse logarithmic relationships of the transcendental numbers PI and E resolve this problem and create the absolute metric without any artifacts and without measurements at all. It suffices to postulate the number PI as the universal unit of space and the number $\mathrm{E}$ as the universal unit of time. Then the first derivative of PI by E can be considered as a unit of speed, and the second derivative is the unit of acceleration. Geometrically, PI is the perimeter; $\mathrm{E}$ is the diameter of the space-time sphere. $\mathrm{E} / 2$ is the radius, $4 * \mathrm{PI}^{*}(\mathrm{E} / 2)^{\wedge} 2$ is the surface of the sphere, and $4 / 3^{*} \mathrm{PI}^{*}(\mathrm{E} / 2)^{\wedge} 3$ is the volume of the sphere, $\mathrm{PI}^{*}(\mathrm{E} / 2)^{\wedge} 2$ is the surface of a circle, $\mathrm{N}^{*}\left(\mathrm{PI}^{*} \mathrm{E}^{\wedge} 2\right) / 4$ is a discrete set of the cylinder volumes. A functional relationships between these space-time geometric parameters and an infinite set of integers $\mathrm{N}$ constitute the basis of the natural [E...PI] binary calculations of the parameters of wave motion [1-5].

The basic principles of universal arithmetic and geometry were developed by Newton, Leibniz and Euler at the beginning of the eighteenth century. The principles led to the creation of the differential calculus and analysis: Unit 1 generates a natural set: $1+1+1+\ldots=1 \ldots$ $(\mathrm{N}+1)$. There is no absolute infinity. Unit 1 and a natural set generate the inverse natural set: $1 /(1+1) \ldots 1 /(\mathrm{N}+1)$. There is no absolute zero. Unit 1 is a quantum of the progressive natural set. Units $1 /(\mathrm{N}+1)$ are the quanta of the inverse natural set. An inverse natural set generates a universal spatial unit PI $=(1+1)^{*} \operatorname{Sum}\{\mathrm{d}(1 / \mathrm{N}) / \operatorname{Sqrt}(1-$ $\left.\left.1 / \mathrm{N}^{\wedge} 2\right)\right\}$ as a circle perimeter. An inverse natural set generates a universal temporal unit $\mathrm{E}=(1+1 / \mathrm{N})^{\wedge} \mathrm{N}$ as the base of natural logarithms.
The modern science has emerged after the discovery of logarithms. But it was impossible to resolve the fundamental problems of infinitesimal calculus before the discovery of the laws of thermodynamics and electrodynamics, from which an approximate scaling unit of temperature $\mathrm{K}$ (kelvin) and a scaling unit of the rotational speed $\mathrm{C}$ (the speed of light) were obtained. It will be shown below that $\mathrm{K}$ and $\mathrm{C}$ are essentially fundamental mathematical units of the inverse natural calculus, such as PI and E of the standard analysis, and they can be obtained analytically from the mutual functional relations of the transcendental numbers PI and E. As the result, all other constants of quantum physics can also be obtained analytically, and their physical meanings can be explained geometrically.

\section{Arithmetical limits of natural computations}

Any two numbers can be a basis of the calculation system, for example binary [0...1], decimal [0...9], quantum binary $[0.000>\ldots 1.111>]$ or natural binary [E...PI]. The functional relations of two transcendental numbers $\mathrm{PI}$ and $\mathrm{E}$ with three natural numbers $\mathrm{A}=137$ (Gaussian prime), $\mathrm{B}=602214183$ (Avogadro's integer) and $\mathrm{R}=105456978$ (integer of Dirac) completely describe a harmonic motion of spherical waves and create the system of natural computations in relative space-time coordinates.

A square of the normalized nine-digit qubit $[1.11111111]^{\wedge} 2=1.2345678987654321$ has a 17-bit mirror symmetry.

A square of the normalized infinite qubit $[1.11111111 \ldots]^{\wedge} 2=1.23456790123456790$ has a 9-bit progressive symmetry. 
A cube of the normalized infinite qubit connects fields of irrational and rational numbers by truncated 17- bit sequence:

The relation $1 / \mathrm{A}=\left(10^{\wedge} 0\right) / 137=$ Sum $\left\{[729927] / 10^{\wedge}\left(8^{*} \mathrm{~N}\right)\right\}$ is the first hyperbolic point of the periodic mirror supersymmetry of the inverse natural set. For any decimal digit a, b, c, d, the ratio [abcdabcd] / $[\mathrm{abcd}]=10001=137 * 73=137 *(137-64)$.

At the same time, the number $A=137$ is very close to the squared sum of root mean the square, arithmetical, geometrical, harmonic mean of PI and $\mathrm{E}$ :

$\left[\operatorname{Sqrt}\left(\left(\mathrm{PI}^{\wedge} 2+\mathrm{E}^{\wedge} 2\right) / 2\right)+(\mathrm{PI}+\mathrm{E}) / 2+\operatorname{Sqrt}(\mathrm{PI} * \mathrm{E})+2 * \mathrm{PI} * \mathrm{E} /(\mathrm{P}\right.$ $\mathrm{I}+\mathrm{E})]^{\wedge} 2=136.9938985020083597=$ SMS.

SMS sequence can be treated as the entropy bound of the parabolic coupling of binary, decimal and natural bit

TRUNCATED PI

3.1415926535897932
3.141592653589793
3.14159265358979
3.1415926535897
3.141592653589
3.14159265358
3.1415926535
3.141592653
3.14159265
3.1415926
3.141592
3.14159
3.1415
3.141
3.14
3.1
3

TRUNCATED E

2.7182818284590452
2.718281828459045
2.71828182845904
2.7182818284590
2.718281828459
2.71828182845
2.7182818284
2.718281828
2.71828182
2.7182818
2.718281
2.71828
2.7182
2.718
2.71
2.7
2

computation systems. The approximate equations $\mathrm{E}^{\wedge} 137=100 * \mathrm{PI}^{*} 10^{\wedge} 57$ and $\mathrm{E}^{\wedge} 137=100 * \operatorname{Sqrt}(10)^{*} 10^{\wedge} 57$ can be treated as the exponential entropy bounds of polynomial coupling of binary, decimal and natural calculation systems:

$100 * \mathrm{PI}^{*} 10^{\wedge} 57=\mathrm{E}^{\wedge} 136.9972503724980956$,

$\mathrm{A}^{\wedge} 1=137.0000000000000000$

$100 * \operatorname{Sqrt}(10) * 10^{\wedge} 57=\mathrm{E}^{\wedge} 137.0038130331457184$.

The computational links of the finite natural difference [PI-E], of the finite natural differential ND = $[\mathrm{PI}-\mathrm{E}] *[\mathrm{PI} / \mathrm{E}] *[\mathrm{E} / \mathrm{PI}]$ and of the finite natural median NM $=\operatorname{Sqrt}(\mathrm{SMS}) / 4$ are shown below:

64-BIT ND

17-BIT NM

$0.4233108251307479 \ldots 999$

$0.4233108251307479 \ldots 999$

$0.4233108251307499 \ldots 999$

$0.4233108251306999 \ldots 999$

$0.4233108251299999 \ldots 999$

$0.4233108251299999 \ldots 999$

$0.4233108250999999 \ldots 998$

$0.4233108249999999 \ldots 999$

$0.4233108299999999 \ldots 999$

$0.4233107999999999 \ldots 999$

$0.4233109999999999 \ldots 999$

$0.4233100000000000 \ldots 000$

0.42329 $99999999999 . .999$

$0.4229999999999999 \ldots 999$

$0.4299999999999999 \ldots 999$

$0.4000000000000000 \ldots 000$

$1.0000000000000000 \ldots 000$

\begin{abstract}
2.9261098161852234
2.9261098161852232

2.9261098161852192

2.9261098161851550

2.9261098161848172

2.9261098161758055

2.9261098161112635

2.9261098156624848

2.9261098100647471

2.9261097755621123

2.9261090710239372

2.9261075871661980

2.9260226569083487

2.9256776292410519

2.9210438087537571

2.8965476238998343

2.4747498748948926
\end{abstract}

The 6-bit integer 42331 and the 16-bit integer 4233108251307479 are prime numbers. This fact limits an accuracy of computations of the hyperbolic functions Cosh and Sinh in the decimal calculation system with floating point.

The characteristic feature of natural differentials (ND) is an appearance of very long sets of digits 9 . It is obvious that $0.9999 \ldots=1$ and $9.999 \ldots=10$. An infinite sequence of 9 is generated as the integer $\mathrm{B}=602214183$ and the integral of Avogadro as $\mathrm{BS}=$ Sum $\left\{\mathrm{B} / 10^{\wedge}\left(3^{*} \mathrm{~N}+11\right)\right\}=0.0060281699999999=0.00602817$.

The characteristic feature of natural medians (NM) is an appearance of the "golden sequence" 1618 over 12-bit [PI...E]. This fact limits an accuracy of the natural median computations of harmonic functions Cos and Sin in decimal system with floating point.
It is easy to show that the use of symbolic infinite (pi...e) and truncated decimal [PI...E] notations leads to essentially different arithmetic results of calculations of polynomial and exponential functions:

$1 / \mathrm{pi}^{\wedge} 32=1 / \mathrm{e}^{\wedge} 37=1 / 10^{\wedge} 16$,

$1 / \mathrm{pi}^{\wedge} 33=1 / \mathrm{e}^{\wedge} 38=0$.

$1 / \mathrm{PI}^{\wedge} 32=1234 / 10^{\wedge} 20>1 / \mathrm{E}^{\wedge} 37=853 / 10^{\wedge} 20$,

$1 / \mathrm{PI}^{\wedge} 33=393 / 10^{\wedge} 20>1 / \mathrm{E}^{\wedge} 38=314 / 10^{\wedge} 20$.

$1 / \mathrm{PI}^{\wedge} 129=1 / \mathrm{E}^{\wedge} 148=1 / \mathrm{NM}^{\wedge} 137=1 / 10^{\wedge} 64$,

$1 / \mathrm{PI}^{\wedge} 130=1 / \mathrm{E}^{\wedge} 149=1 / \mathrm{NM}^{\wedge} 138=0$.

$\mathrm{e}^{\wedge}\left(\mathrm{j}^{*}\right.$ pi) $+1=0$ in symbolic (pi...e) continuous mathematics,

$E^{\wedge}\left(j^{*} P I\right)+1=1 / 10^{\wedge} 64>0$ in binary $[0 \ldots 1]$ discrete mathematics,

$\mathrm{E}^{\wedge}\left(\mathrm{j}^{*} \mathrm{PI}\right)+1$ is about $[1 / 137] / 10^{\wedge} 57>0$ in qubit $[0>\ldots 1>]$ arithmetic. 


\section{Natural quantum metric}

Seven scaling units are sufficient to constitute the natural metric system. The numbers PI and E are the basis of natural differential computing, the numbers $\mathrm{C}$ and $\mathrm{K}$ are the basis of a natural median computing. The integers $\mathrm{R}$, A, B mutually co-ordinate the natural differentials (ND) and the natural medians $(\mathrm{NM})$ :

$\mathrm{PI}=(1+1) * \operatorname{Sum}\left\{\mathrm{d}(1 / \mathrm{N}) / \operatorname{Sqrt}\left(1-1 / \mathrm{N}^{\wedge} 2\right)\right\}=$

3.1415926535897932 (spatial unit of Pythagoras), $\mathrm{E}=\left(1+1 / \mathrm{N}^{\wedge} \mathrm{N}\right)^{\wedge}\left(\mathrm{N}^{\wedge} \mathrm{N}\right)=2.7182818284590452$

(temporal unit of Euler),

$\mathrm{C}=\left(\mathrm{R} / 10^{\wedge} 8+4 * \mathrm{PI} \mathrm{C}^{*} / 10^{\wedge} 18\right)^{\wedge} 64 * 10^{\wedge} 7=$

299792457.86759134 (speed unit of Maxwell),

$\mathrm{K}=\mathrm{E}+\mathrm{AS}+\mathrm{BS}=2.7315999984590452 \quad$ (temperature unit of Kelvin),

$\mathrm{R} / 10^{\wedge} 8=\operatorname{Integer}\left\{10^{\wedge} 8^{*}\left(\mathrm{C} / 10^{\wedge} 7\right)^{\wedge}(1 / 64)\right\} / 10^{\wedge} 8=$

1.0545697800000000 (radial unit of Dirac),

$\mathrm{AS}=1 / 100 / \operatorname{Sum}\left\{[\mathrm{A}+(\mathrm{A}-100) * \mathrm{~N}] / 10^{\wedge}(3 * \mathrm{~N}+2)\right\}=$ 0.0072900000000000 (eccentricity unit of Sommerfeld),

$\mathrm{BS}=\operatorname{Sum}\left\{\mathrm{B} / 10^{\wedge}\left(3^{*} \mathrm{~N}+11\right)\right\}=0.0060281699999999$ (entropy unit of Avogadro).

\section{Matrix equations of wave motion}

Ten matrix equations of the differential geometry in a topologically inverted space-time determine parameters of the push-pull spherical wave motion:

$[\mathrm{R}]=1+2 / 100 *(\mathrm{E}+[\mathrm{A}] *(1+\operatorname{Sqrt}(2 * \mathrm{PI} \mathrm{E} / 100)))$ - matrix field of the normalized inverse radius (Dirac's units).

$[\mathrm{A}]=(100 *([\mathrm{R}]-1) / 2-\mathrm{E}) /(1+\operatorname{Sqrt}(2 * \mathrm{PI} * \mathrm{E} / 100))$ matrix field of the normalized inverse eccentricity (Sommerfeld's units).
[N]
[A]
0.0060221410025819
0.0073189621138002
[R]
$0.0060221410053903 \quad 0.0073187289405399$
$0.0060221410732354 \quad 0.0073131309589000$
$0.0060221411450152 \quad 0.0073071361524362$
$\begin{array}{lll}.0545725104198716 & 1.6605390002845580\end{array}$
$1.0545725038291215 \quad 1.6605389995101736$
$\begin{array}{lll}1.0545723455995802 & 1.6605389808026354\end{array}$
$1.0545721761536017 \quad 1.6605389610101475$

$[\mathrm{P}]=2 * \mathrm{PI} * \mathrm{R}]-$ matrix field of the normalized inverse perimeter (Planck's units).

$[\mathrm{G}]=[\mathrm{P}]^{*}(1+[\mathrm{A}])-$ matrix field of the normalised density of inverse perimeters (Newton's gravity units).

$[\mathrm{V}]=[\mathrm{R}]^{\wedge} 64^{*} 10^{\wedge} 7$ - matrix field of the relative speed of waves (Maxwell's units).

$[\mathrm{MM}]=12-[\mathrm{A}] / 10$ - matrix field of the relative amplitude of waves (Amagat's molar concentration units).

$[\mathrm{KB}]=\operatorname{Cos}[\mathrm{MM}]-\operatorname{Sin}[\mathrm{MM}]-$ matrix field of the relative phase of waves (Boltzmann's units).

$[\mathrm{W}]=[\mathrm{V}]^{\wedge} 2-$ matrix field of the relative energy of waves (Einstein's units).

$[\mathrm{NA}]=\left\{\operatorname{Sqrt}\left(8^{*} \mathrm{PI} * \mathrm{E} /\left(8^{*} \mathrm{PI} * \mathrm{E}+137^{\wedge} 2\right)\right) /(1+2 *[\mathrm{~A}] / 1000)-\right.$ $\left.5 / 10^{\wedge} 8\right\} / 10$ - field of Avogadro entropy units.

$[\mathrm{DA}]=1 /[\mathrm{NA}] / 100$ - normalized inverse information entropy (Dalton's atomic mass units).

\section{Matrix of quantum invariants}

The arithmetical entropy matrix of Avogadro-Dalton: $\mathrm{A} 4=(\mathrm{PI} * \mathrm{E} / 100)^{\wedge} 2+\left(1 / 137-(\mathrm{PI} * \mathrm{E} / 100)^{\wedge} 2\right.$ is upper parabolic bound of the inverse orbital eccentricity. $\mathrm{AH}=1 /\left(16^{*} \mathrm{PI}^{*} \mathrm{E}\right)$ is upper hyperbolic bound of the inverse orbital eccentricity.

$\mathrm{AB}=\mathrm{A}(\mathrm{NB}), \mathrm{NB}=602214183 /\left(1+4 * \mathrm{PI} / 10^{\wedge} 8\right) / 10^{\wedge} 11$ is PI-reference point of the information entropy of eccentricity.

$\mathrm{AL}=1 /\left(1+59^{*} \operatorname{Ln}(10)\right)$ is upper logarithmic bound of inverse orbital eccentricity.
The geometrical entropy matrix of PlanckSommerfeld:

$A 1=1 / 137$ is central point of natural mirror symmetry of inverse orbital eccentricity. AF $=1000 /$ Integer $\{1000 *$ Sqrt $\left.\left(137^{\wedge} 2+\mathrm{PI}^{\wedge} 2\right)\right\}$ is PI-reference point of inverse orbital eccentricity.
[N]
[A]
$0.0060221412397551 \quad 0.0072992700729927$
[R]
$0.0060221412628504 \quad 0.0072973525205056$
$\begin{array}{lll}0.0060221413188129 & 0.0072927060593902\end{array}$
$0.0060221413514050 \quad 0.0072900000000000$
$\begin{array}{lll}1.0545719538152265 & 6.6260710055755005\end{array}$
$\begin{array}{ll}1.0545718996147182 & 6.6260706650236630\end{array}$
$\begin{array}{lll}1.0545717682803448 & 6.6260698398254579\end{array}$
$1.0545716917923240 \quad 6.6260693592370495$ orbital eccentricity. bound of orbital eccentricity.

AS $=1 / 100 /(1.111111111>)^{\wedge} 3$ is lower hyperbolic Maxwell

The differential entropy matrix of Kelvin-

$\mathrm{RC}=\left(\mathrm{R}+4^{*} \mathrm{PI}{ }^{*} \mathrm{C} / 10^{\wedge} 10\right) / 10^{\wedge} 8$ is the PI-reference point of core radius. $\mathrm{RE}=(\mathrm{R}+1 / \mathrm{E}) / 10^{\wedge} 8$ is the $\mathrm{E}$ reference point of core radius.
$\mathrm{RA}=(\mathrm{R}+1 /(\mathrm{E}+\mathrm{AS})) / 10^{\wedge} 8$ is the $\mathrm{A}-\mathrm{E}$ reference point of core radius.

$\mathrm{RK}=\left(\mathrm{R}+1 /(\mathrm{E}+\mathrm{AS}+\mathrm{BS}) / 10^{\wedge} 8\right.$ is the K-reference point of core radius.
[KB]
[A]
[R]
[V]
$\begin{array}{llllll}1.3806484502840000 & 0.0072224962396475 & 1.0545697837673031 & 299792457.86759134\end{array}$
$\begin{array}{lllll}1.3806484501880000 & 0.0072224931083101 & 1.0545697836787944 & 299792456.08618159\end{array}$
$\begin{array}{lllll}1.3806484501770000 & 0.0072224927601997 & 1.0545697836689549 & 299792456.07825451\end{array}$ 


\section{$\begin{array}{llll}1.3806484501680000 & 0.0072224924737441 & 1.0545697836608581 & 299792455.93094320\end{array}$}

The median point of a normal distribution of the Wien wavelength displacement:

\section{$[\mathrm{NX}]$ \\ $0.0060221445289294 \quad 0.0070261763632109$ \\ 6 Primary physical elements of matter}

$\mathrm{AX}=5 / \operatorname{Root}\left\{\mathrm{X}^{*} \mathrm{E}^{\wedge} \mathrm{X} /\left(\mathrm{E}^{\wedge} \mathrm{X}-1\right)=5\right\}-1$ is the lower bound of the relative inverse eccentricity:

[RX] [GX] $1.0545642346951568 \quad 6.6725781076198224$
Background tempo of the core waves: $\mathrm{E}+\mathrm{AS}+\mathrm{BS}=2.7315999984590452=\mathrm{K}$ is the upper bound of the background tempo.

Med $\{$ EA...K $\}=2.7285850810946404$.

$\mathrm{E}+\mathrm{AS}=2.7255718284590452=\mathrm{EA}$.

Med of meds $=2.7252543275634558=$ TBG is the median background tempo.

Med $\{$ EA...E $\}=2.7219256081809303$.

$\mathrm{E}+0=2.7182818284590452=\mathrm{E}$ is the lower bound of the background tempo.

Vibrational speed of the core waves:

$\mathrm{RE}^{\wedge} 64 * 10^{\wedge} 7=2.9979245625727419^{*} 10^{\wedge} 8=\mathrm{TE}$.

Med $\{$ TA...TE $\}=2.9979245616776434 * 10^{\wedge} 8$.

Med of meds $=2.9979245608618159^{*} 10^{\wedge} 8=\mathrm{TN}+$.

Med $\{\mathrm{TN}-\ldots \mathrm{TN}+\}=2.9979245608610649 * 10^{\wedge} 8=\mathrm{T} 0$ is the median vibrational speed.

$\left.\mathrm{R}+1 / \mathrm{TBG} / 10^{\wedge} 8\right)^{\wedge} 64 * 10^{\wedge} 7=2.9979245608603140^{*} 10^{\wedge} 8$ $=\mathrm{TN}$.

$\mathrm{RA}^{\wedge} 64 * 10^{\wedge} 7=2.9979245607825451 * 10^{\wedge} 8=\mathrm{TA}$.

Med $\{$ TA...TK $\}=2.9979245600459885^{*} 10^{\wedge} 8$.

$\mathrm{RK}^{\wedge} 64 * 10^{\wedge} 7=2.9979245593094320^{*} 10^{\wedge} 8=\mathrm{TK}$.

Rotational speed of the core waves:

$\left.\mathrm{CN}+=\mathrm{R} / 10^{\wedge} 8+4 * \mathrm{PI} \mathrm{C}^{*} / 10^{\wedge} 18\right)^{\wedge} 64 / 10=$ $2.9979245786759134 * 10^{\wedge} 8$.

Med $\{\mathrm{CN}-\ldots \mathrm{CN}+\}=2.9979245786759103^{*} 10^{\wedge} 8=\mathrm{C} 0$ is the median rotational speed.

$\mathrm{CN}-=\mathrm{RC}^{\wedge} 64 / 10=2.9979245786759074 * 10^{\wedge} 8$.

Translational velocity of the core waves:

Med $\{\mathrm{T} 0 \ldots \mathrm{C} 0\}=2.9979245697684876^{*} 10^{\wedge} 8=\mathrm{V} 0$ is the median translational velocity.

Median radius of the core waves:

$\left(\mathrm{V} 0 / 10^{\wedge} 7\right)^{\wedge}(1 / 64)=1.0545697837183448=\mathrm{RV} 0$.

Median eccentricity of the core waves:

$(100 *(\mathrm{RV} 0-1) / 2-\mathrm{e}) /(1+\operatorname{Sqrt}(2 * \mathrm{PI} * \mathrm{E} / 100))$

$0.0072224945075574=$ AV0.

Relative amplitude of the core waves:

12-AV0/10 = $11.9992777505492443=\mathrm{MM}$ (molar mass)

Relative phase of the core waves:

$\operatorname{Cos}(\mathrm{MM})-\operatorname{Sin}(\mathrm{MM})=1.3806484502310000=\mathrm{KB}$

(Boltzmann's unit).

Density of the nuclear waves:

$2 *$ PS $*(1+$ AS $)=6.6743734048658876=$ GS

Med $\{$ GV0...GS $\}=6.6741437171665912$

Med of meds $=6.6736948779387962=\mathrm{GM}$ is the lower bound of the gravitational unit.

$\mathrm{GV0}=2 * \mathrm{PI} * \mathrm{RV0} *(1+\mathrm{AV} 0)=6.6739140334195273$

Med $\{\mathrm{GV0} \ldots \mathrm{GX}\}=6.6732460538038305$

Mechanical energy quanta $\mathrm{EM}=\left(\mathrm{m}^{*} \mathrm{v}^{\wedge} 2 / 2\right)^{*}\left[1+\left(\mathrm{v}^{\wedge} 2\right) /(\mathrm{T} 0 \ldots \mathrm{C} 0)^{\wedge} 2\right]$, (from Newton to Einstein).

Thermal energy quanta

Electrical energy quanta

Optical energy quanta
$2 * \mathrm{PX}^{*}(1+\mathrm{AX})=6.6725781076198224=\mathrm{GX}$

Parameters of the orbital waves:

$\mathrm{R} 1=1.0545719538152265,2 * \mathrm{PI} \mathrm{R} 1=$ $6.6260710055755005=\mathrm{P} 1$.

Med $\{\mathrm{P} 0 \ldots \mathrm{P} 1\}=6.6260704227004664$.

Med of meds $=6.6260700111158524=\mathrm{PP}$ is the median perimeter (Planck's unit).

$\mathrm{R} 0=1.0545717682803448, \quad 2 * \mathrm{PI} * \mathrm{R} 0=$ $6.6260698398254579=\mathrm{P} 0$.

Med $\{\mathrm{P} 0 \ldots \mathrm{PS}\}=6.6260695995312515$.

$\mathrm{RS}=1.0545716917923240, \quad 2 * \mathrm{PI} * \mathrm{RS}=$ $6.6260693592370495=$ PS.

$\mathrm{PP} /(2 * \mathrm{PI})=1.0545717955420578=\mathrm{RP}$ is the reduced Planck unit.

$(100 *(\mathrm{RP}-1) / 2-\mathrm{E}) /(1+\operatorname{Sqrt}(2 * \mathrm{PI} * \mathrm{E} / 100))=$ $0.0072936705478647=\mathrm{AP}$.

$1000 /$ Integer $\left\{1000^{*} \operatorname{Sqrt}\left(137^{\wedge} 2+\mathrm{PI}^{\wedge} 2\right)\right\}$

$0.0072973525205056=\mathrm{AF}$ is the fine structure constant. $1+2 / 100 *(\mathrm{E}+\mathrm{AF} *(1+\operatorname{Sqrt}(2 * \mathrm{PI} * \mathrm{E} / 100)))$

$1.0545718996147182=\mathrm{RF}$.

The median density of the orbital waves

Med $\{$ RP...RF $\}=1.0545718475783874=\mathrm{RQ}$.

$(100 *(\mathrm{RQ}-1) / 2-\mathrm{E}) /\left(1+\operatorname{Sqrt}\left(2 * \mathrm{PI} \mathrm{E}^{*} / 100\right)\right)$

$0.0072955115341644=\mathrm{AQ}$.

$2 * \mathrm{PI} * \mathrm{RQ}=6.6260703380697541=\mathrm{PQ}$.

$\mathrm{PQ}^{*}(1+\mathrm{AQ})=6.6744109106473266=\mathrm{GQ}$ is the upper bound of the gravitation unit.

The median orbital-nuclear density of waves

Med (GM...GQ) $=6.6740528894917918=\mathrm{G}$ is $\mathrm{a}$ median of the Newtonian gravitational unit.

The elementary charge unit:

$\operatorname{Sqrt}\left(10 * \mathrm{AF}^{*} \mathrm{RP} / \mathrm{V} 0\right)=1.6021766150249783=\mathrm{Q}$ is the Stoney elementary charge unit.

The median information entropy:

$$
\mathrm{N}(\mathrm{A} 4)=0.0060221410025819=\mathrm{N} 4 \text {. }
$$

Med $\{$ NH...N4 $\}=0.0060221410039861=$ NH4.

$\mathrm{N}(\mathrm{AH})=0.0060221410053903=\mathrm{NH}$.

Med $\{$ NHL...NH4 $\}=0.0060221410395944=$ NE.

Med $\{$ NB...NE $\}=0.0060221410564149=\mathrm{NA}$ is the median of the Avogadro entropy unit.

$$
\mathrm{NB}=0.0060221410732354 \text {. }
$$

Med $\{$ NH...NL $\}=0.0060221410752027=$ NHL. $\mathrm{N}(\mathrm{AL})=0.0060221411450152=\mathrm{NL}$

Decimal orders of quantum units are easily extracted from the gauge equation $\mathrm{E}^{\wedge} 137=\mathrm{PI}^{*} 10^{\wedge} 59$ and from the Wien wavelength displacement law $\mathrm{WD}=$ $2 * \mathrm{PI} * \mathrm{RP} *(\mathrm{~T} 0 \ldots \mathrm{C} 0) /\left(\mathrm{KB}^{*} \mathrm{X}\right)$. A natural computing fully coordinates classical and quantum physics: $\mathrm{ET}=\left(\mathrm{k}^{*} \mathrm{~T}\right) /\left[1+\left(\mathrm{v}^{\wedge} 2\right) /(\mathrm{T} 0 \ldots \mathrm{C} 0)^{\wedge} 2\right],($ from Kelvin to Boltzmann $)$. $\mathrm{EE}=\left(\mathrm{q}^{*} \mathrm{U}\right) /\left[1+\left(\mathrm{v}^{\wedge} 2\right) /(\mathrm{T} 0 \ldots \mathrm{C} 0)^{\wedge} 2\right]$, (from Stoney to Dirac). $\mathrm{EA}=\left(\mathrm{h}^{*} \mathrm{f}\right) *\left[1+\left(\mathrm{v}^{\wedge} 2\right) /(\mathrm{T} 0 \ldots \mathrm{C} 0)^{\wedge} 2\right],($ from Planck to Shannon). 


\section{References}

1. Eugene Machusky, Quantum Metric of Classic Physics,

(2017),

iopscience.iop.org/article/10.1088/1757899X/239/1/012009.

2. Eugene Machusky, Unified Quantum Metric, (2017), J Laser Opt Photonics 4:3 (Suppl) DOI:10.4172/2469-410X-C1-014.

3. Eugene Machusky, Quantum Physics as Dynamic Space-Time Differential Calculus, (2017), J Material Sci Eng 6:7 (Suppl) DOI:10.4172/2
4. Eugene Machusky, Quantum Physics as Dynamic Space-Time Differential Calculus, review article (2017), Adv. Material Sci Engg, volume 1, issue 1,1 of 4 . http://www.opastonline.com/wpcontent/uploads/2017/12/quantum-physics-asdynamic-space-time-differential-calculus-amse$17 . p d f$

5. Eugene Machusky, Complex Geometry of Wave Motion, (2018), Int J Eng Tech DOI: 10.7763/IJET.2018.v10.1056. 